Research Article

\title{
Functionally Separation Axioms on General Topology
}

\author{
Abdelwaheb Mhemdi $\mathbb{D}^{1}$ and Tareq M. Al-shami $\mathbb{D D}^{2}$ \\ ${ }^{1}$ Department of Mathematics, College of Sciences and Humanities in Aflaj, Prince Sattam Bin Abdulaziz University, \\ Riyadh, Saudi Arabia \\ ${ }^{2}$ Department of Mathematics, Sana'a University, Sana'a, Yemen
}

Correspondence should be addressed to Abdelwaheb Mhemdi; mhemdiabd@gmail.com

Received 18 January 2021; Accepted 23 April 2021; Published 13 May 2021

Academic Editor: Jia-Bao Liu

Copyright (C) 2021 Abdelwaheb Mhemdi and Tareq M. Al-shami. This is an open access article distributed under the Creative Commons Attribution License, which permits unrestricted use, distribution, and reproduction in any medium, provided the original work is properly cited.

In this paper, we define a new family of separation axioms in the classical topology called functionally $T_{i}$ spaces for $i=0,1,2$. With the assistant of illustrative examples, we reveal the relationships between them as well as their relationship with $T_{i}$ spaces for $i=0,1,2$. We demonstrate that functionally $T_{i}$ spaces are preserved under product spaces, and they are topological and hereditary properties. Moreover, we show that the class of each one of them represents a transitive relation and obtain some interesting results under some conditions such as discrete and Sierpinski spaces.

\section{Introduction and Preliminaries}

Topology is a branch of mathematics that researches properties of spaces that are invariant under any continuous deformation. That is, it forms a novel kind of geometry that relies on nearness or neighborhood of elements instead of measuring distance between them. Recently, topological spaces have been applied to model practical problems, especially those related to information system; see, for example [1-3].

Separation axioms are one among the most common, important, and interesting ideas in topology. They can be used to initiate different classes of topological spaces and determine the type of some subsets. Some separation axioms were defined using continuous maps (see, [4-6]) on one hand. On the other hand, some separation axioms were introduced using generalized open sets (see [7] and the references mentioned therein). Also, separation axioms have been generalized to other spaces in general topology like pretopological spaces, ordered topological spaces, and generalized topological spaces (see [8-11]). Another generalization of separation axioms was given using functions in the category of topological spaces (see [12]). We draw attention to some separation axioms have been very useful in the study of certain objects in digital topology (see $[13,14]$ ).
This work is organized as follows: after this introduction, we recall some definitions and results which are required to make this work self-contained. In Section 2, we introduce the concepts of functionally $T_{i}$ space for $i=0,1,2$. We study their main properties, especially those are related to product spaces and topological and hereditary properties. In Section 3 , we explore some findings that associated these concepts with the discrete and Sierpinski spaces. We write some conclusions and propose some future works in Section 4 .

The following definitions are mentioned in [15].

Definition 1. A subcollection $\tau$ of $2^{X}$ is called a topology on $X$ provided that it is closed under arbitrary union and finite intersection. A pair $(X, \tau)$ is called a topological space. Every subset of $X$ belong to $\tau$ is called an open set, and its complement is called a closed set.

Definition 2. A bijective map $f:(X, \tau) \longrightarrow(Y, \theta)$ is said to be a homeomorphism if it is continuous (i.e., the inverse image of every open (resp., closed) set is open (resp., closed)) and open (i.e., the image of every open set is open).

A property which is preserved by every homeomorphism map is called a topological property. 
Definition 3. Let $A$ be a set in a topological space $(X, \tau)$. The family $\tau_{A}=\{A \cap G: G \in \tau\}$ is called a relative topology on $A$. A pair $\left(A, \tau_{A}\right)$ is called a subspace of $(X, \tau)$.

A property which is preserved by every subspace is called a hereditary property.

Definition 4. $(X, \tau)$ is said to be as follows:

(1) $T_{0}$ provided that, for every $a \neq b \in X$, there exists an open set containing only one of them

(2) $T_{1}$ provided that, for every $a \neq b \in X$, there exist two open sets such that one containing $a$ but not $b$ and the other containing $b$ but not $a$

(3) $T_{2}$ (or Hausdorff) provided that there exist two disjoint open sets $U$ and $V$ for every $a \neq b \in X$ such that $a \in U$ and $b \in V$

Proposition 1. For any two sets $A$ and $B$, we have $(G \times H) \cap(U \times V)=(G \cap H) \times(U \cap V)$.

Definition 5. Let $\left\{\left(X_{i}, \tau_{i}\right): i=1,2, \ldots, n\right\}$ be the collection of topological spaces. Then, $\mathscr{B}=\prod_{i=1}^{n} \tau_{i}=\left\{\prod_{i=1}^{n} G_{i}: G_{i} \in \tau_{i}\right\}$ defines a base for a topology $\tau$ on $X=\prod_{i=1}^{n} X_{i}$. We call $(X, \tau)$ a finite product space.

Definition 6. A binary relation $\rho$ on $X$ (i.e., $\rho \subseteq X \times X)$ is said to be transitive if $(x, z) \in \rho$ whenever $(x, y) \in \rho$ and $(y, z) \in \rho$.

\section{Relative Separation Axioms}

In this portion, we formulate the concepts of functionally $T_{i}$ space $(i=0,1,2)$ using continuous maps. We explore their basic properties and provide some interesting examples to clarify the presented results.

Definition 7. Let $(X, \tau),(Y, \theta)$ be two topological spaces. $(X, \tau)$ is said to be as follows:

(1) A functionally $T_{0}$ space relatively to $(Y, \theta)$ or, for short, $X$ is $F T_{(0, Y)}$ if for any distinct points $x, y \in X$, there exist a continuous map $f$ from $X$ to $Y$ and $U \in \theta \quad$ such that $\{f(x), f(y)\} \cap U \in\{\{f(x)\},\{f(y)\}\}$

(2) A functionally $T_{1}$ space relatively to $(Y, \theta)$ or, for short, $X$ is $F T_{(1, Y)}$ if for any distinct points $x, y \in X$, there exist a continuous map $f$ from $X$ to $Y$ and $U, V \in \theta$ such that $f(x) \in(U / V)$ and $f(y) \in(V / U)$

(3) A functionally $T_{2}$ space relatively to $(Y, \theta)$ or, for short, $X$ is $F T_{(2, Y)}$ if for any distinct points $x, y \in X$, there exist a continuous map $f$ from $X$ to $Y$ and $U, V \in \theta$ such that $U \cap V=\varnothing, \quad f(x) \in U$ and $f(y) \in V$

\section{Remark 1}

(1) If $X$ is a singleton, then for any topological space $(Y, \theta), X$ is $F T_{(i, Y)}$ for each $i=0,1,2$
(2) If $(Y, \theta)$ is the indiscrete topology and $|X| \geq 2$, then $(X, \tau)$ is not $F T_{(0, Y)}$ for each topology $\tau$ on $X$

Proposition 2. $(X, \tau)$ is a $T_{i}$-space if and only if it is $F T_{(i, X)}$ for each $i=0,1,2$.

Proof. It is sufficient to take the identity map id from $(X, \tau)$ to itself. It is clear that $i d$ is a continuous map satisfying the desired result.

It is easy to prove the following implications.

Proposition 3. Every $F T_{(i, Y)}$ space is $F T_{(i-1, Y)}$ for each $i=1,2$.

We give the following example to clarify the fail of the converse of Proposition 3.

Example 1. Let $\tau_{1}=\{\varnothing, X,\{a\}, t\{a, b\}\}$ be a topology on $X=$ $\{a, b, c\}$ and $\tau_{2}$ be the cofinite topology on the set of natural numbers $\mathbb{N}$. It is clear that $\left(X, \tau_{1}\right)$ and $\left(\mathbb{N}, \tau_{2}\right)$ are, respectively, $T_{0}$ and $T_{1}$ spaces. On the other hand, $\left(X, \tau_{1}\right)$ is not a $T_{1}$ space and $\left(\mathbb{N}, \tau_{2}\right)$ is not a $T_{2}$ space. Without loss of generality, consider $X=Y$. Then, it follows from Proposition 3 that $\left(X, \tau_{1}\right)$ is $F T_{(0, Y)}$ but not $F T_{(1, Y)}$. Also, consider $Y=\mathbb{N}$. Then, $\left(\mathbb{N}, \tau_{2}\right)$ is $F T_{(1, Y)}$ but not $F T_{(2, Y)}$.

Proposition 4. For all $i \in\{0,1,2\}$, if $(X, \tau)$ is $F T_{(i, Y)}$, then $(X, \tau)$ is a $T_{i}$-space.

Proof. When $i=0$. Suppose that $x \neq y$ in an $F T_{(0, Y)}$-space. Then, there exist a continuous map $f$ from $X$ to $Y$ and $U \in \theta$ such that $\{f(x), f(y)\} \cap U \in\{\{f(x)\},\{f(y)\}\}$. Now, $f^{-1}(U)$ is an open subset of $X$ containing only one of the elements $x$ and $y$ but not both. Hence, $X$ is a $T_{0}$-space.

By analogous it is easy to prove the proposition for $i \in\{1,2\}$.

Corollary 1. If $(X, \tau)$ is a finite $F T_{(1, Y)}$ space, then $\tau$ is the discrete topology.

The converse of the proposition above fails as shown in the following example.

Example 2. Let $\tau$ be the discrete topology on $X=\{a, b, c\}$ and $\theta=\{\varnothing, Y,\{1\}\}$ be a topology on $Y=\{1,2,3,4\}$. It is clear that $(X, \tau)$ is a $T_{2}$ space. On the other hand, let $f:(X, \tau) \longrightarrow(Y, \theta)$ be any continuous map. While $f(a)$, $f(b)$ and $f(c)$ are three distinct points in $(Y, \theta)$, a topology $\theta$ contains only one proper open subset of $Y$; hence, $(X, \tau)$ is not $F T_{(2, Y)}$.

We summarize the previous relationships in Figure 1.

Theorem 1. If $(X, \tau)$ and $(Y, \theta)$ are $T_{i}$ space such that $X$ is finite, then $(X, \tau)$ is an $F T_{(i, Y)}$-space, where $i=1,2$.

Proof. We only prove the theorem in case of $i=1$.

Since $(X, \tau)$ a finite $T_{1}$ space, then every map from $X$ to $Y$ is continuous. Therefore, for every $x \neq y \in X$, we define a 


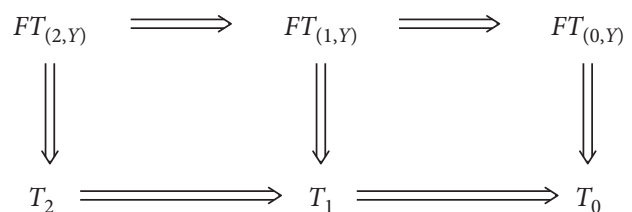

FIGURE 1: The relationships between functionally separation axioms and classical separation axioms.

continuous map $f$ such that $f(x) \neq f(y) \in Y$. Since $(Y, \theta)$ is a $T_{1}$ space, there exist open subsets $U, V$ of $Y$ such that $f(x) \in(U / V)$ and $f(y) \in(V / U)$. Hence, the proof is complete.

In the following result, we investigate under which conditions the converse of Proposition 4 is true.

Proposition 5. Let $g:(X, \tau) \longrightarrow(Y, \theta)$ be an injective bicontinuous map. Then, $(X, \tau)$ is a $F T_{(i, Y)}$ space if and only if $(X, \tau)$ is a $T_{i}$ space for each $i=0,1,2$.

Proof. We only prove the proposition incase of $i=2$. The other cases are proved similarly.

The necessary part follows from Proposition 4 . To prove the sufficient part, let $x \neq y \in X$. By hypothesis, there exist two disjoint open sets $U, V \in \tau$ such that $x \in U$ and $y \in V$. Since $g$ is injective, we obtain $g(x) \neq g(y) \in Y$, and since $g$ is open, then $g(U)$ and $g(V)$ are disjoint open sets in $\theta$ containing $g(x)$ and $g(y)$, respectively. It comes from the continuity of $g$ that $(X, \tau)$ is a $F T_{(2, Y)}$. Hence, we obtain the desired result.

Theorem 2. The finite product of $F T_{(i, Y)}$ spaces is $F T_{(i, Y)}$ for each $i=0,1,2$.

Proof. We only prove the proposition incase of $i=2$.

Let $\left(X_{1}, \tau_{1}\right)$ and $\left(X_{2}, \tau_{2}\right)$ be two $F T_{(2, Y)}$ spaces. Then, for all $a_{1} \neq a_{2} \in X_{1}$, there exists a continuous map $f$ from $X_{1}$ to $Y$ and $F, G \in \tau_{1}$ such that $F \cap G=\varnothing, f\left(a_{1}\right) \in F$ and $f\left(a_{2}\right) \in G$, and for all $b_{1} \neq b_{2} \in X_{2}$, there exists a continuous map $g$ from $X_{2}$ to $Y$ and $U, V \in \tau_{2}$ such that $U \cap V=\varnothing, f\left(b_{1}\right) \in U$ and $f\left(b_{2}\right) \in V$. Suppose that $\left(a_{1}, b_{1}\right) \neq\left(a_{1}, b_{1}\right) \in X_{1} \times X_{2}$. Then, $a_{1} \neq a_{2}$ or $b_{1} \neq b_{2}$. Without loss of generality, consider $a_{1} \neq a_{2}$. Taking a map $h: X_{1} \times X_{2} \longrightarrow Y_{1} \times Y_{2}$ defined by $h(a, b)=(f(a), g(b))$. It can be checked that $h$ is a continuous map. Now, we have $F \times Y_{2}$ is an open subset of $Y_{1} \times Y_{2}$ containing $h\left(a_{1}, b_{1}\right)$ and $G \times Y_{2}$ is an open subset of $Y_{1} \times Y_{2}$ containing $h\left(a_{2}, b_{2}\right)$ such that $\left(F \times Y_{2}\right) \cap\left(G \times Y_{2}\right)=\varnothing$. Hence, $X_{1} \times X_{2}$ is a $F T_{\left(2, Y_{1} \times Y_{2}\right)}$.

By analogous one can prove the other two cases.

Theorem 3. The property of being an $F T_{(i, A)}$ space is a topological property for each $i=0,1,2$.

Proof. We only prove the proposition incase of $i=2$.

Suppose that $f:(X, \tau) \longrightarrow(Y, \theta)$ is a homomorphism map. To prove that $(Y, \theta)$ is a $F T_{(2, A)}$ space, let $x \neq y \in Y$. Then, $f^{-1}(x) \neq f^{-1}(y) \in X$. Since $(X, \tau)$ is a $F T_{(2, A)}$ space, then there exists a continuous map $g$ from $X$ to $A$ and $F, G \in \tau$ such that $F \cap G=\varnothing, \quad g\left(f^{-1}(x)\right) \in F \quad$ and $g\left(f^{-1}(y)\right) \in G$. Since $f$ is a homomorphism and $g$ is continuous, then $g \circ f^{-1}: Y \longrightarrow A$ is a continuous map satisfying that $(Y, \theta)$ is a $F T_{(2, A)}$ space. Hence, the proof is complete.

By analogous one can prove the other two cases.

Theorem 4. The property of being an $F T_{(i, A)}$ space is a hereditary property for each $i=0,1,2$.

Proof. The proof follows from the fact a continuous map $f:(X, \tau) \longrightarrow(Y, \theta)$ implies that the restriction map $f:\left(A, \tau_{A}\right) \longrightarrow(Y, \theta)$ is also continuous for every nonempty subset $A$ of $X$.

Theorem 5. The binary relation defined on the set of topological spaces by $(X, \tau) \sim(Y, \theta)$ if and only if $X$ is $F T_{(i, Y)}$ is transitive.

Proof. Let $(X, \tau),(Y, \theta)$, and $\left(Z, \tau_{Z}\right)$ be three topological spaces such that $(X, \tau) \sim(Y, \theta)$ and $(Y, \theta) \sim\left(Z, \tau_{Z}\right)$. If $x \neq y \in X$, then there exists a continuous map $f$ from $X$ to $Y$ satisfying the conditions given in Definition 2.1 for each $i$. Now, since $f(x) \neq f(y)$, then there exists also a continuous map $g$ from $Y$ to $Z$ satisfying the same conditions, so that the composition $g \circ f$ is a continuous map from $X$ to $Z$ verifying the conditions in the separation $i$ for the points $(g \circ f)(x)$ and $(g \circ f)(y)$. Thus, $(X, \tau) \sim\left(Z, \tau_{Z}\right)$; hence, $\sim$ is transitive.

\section{Properties and Particular Cases}

In this section, we present some properties of $F T_{(i, Y)}$ spaces under some particular of topological spaces such as discrete and Sierpinski spaces.

Proposition 6. Let $(X, \tau)$ and $(Y, \theta)$ be two topological spaces such that $|X|>1$ and $|Y|=2$. If $(X, \tau)$ is $F T_{(1, Y)}$, then $\tau$ and $\theta$ are discrete topologies.

Proof. Let $x \neq y \in X$. Then, there exists a continuous map from $X$ to $Y$ and $U \in \theta$ such that $f(x) \in U$ and $f(y) \notin U$, then $U=\{f(x)\} \in \theta$. Similarly, we prove that $\{f(y)\} \in \theta$. Thus, $\theta=\tau_{d}$. Also, it follows from Corollary 1 that $\tau$ is the discrete topology on $X$.

In the rest of this paper we will denote by $D$ the topological space $\{0,1\}$ with the discrete topology.

Theorem 6. The next statements are identical:

(1) $(X, \tau)$ is $F_{(0, D)}$

(2) For all $x \neq y$, there exists a clopen (open and closed) containing $x$ and not containing $y$

Proof

$(1) \Rightarrow(2)$ : suppose $X$ be $F T_{(0, D)}$. Let $x \neq y \in X$, then there exists $f \in C(X, Y)$ such that $f(x)=0$ and 
$f(y)=1$. So that $f^{-1}(\{f(x)\})$ is a clopen containing $x$ but not containing $y$.

(2) $\Rightarrow(1)$ : let $x \neq y \in X$ and $U$ a open set containing $x$ and not containing $y$. Now, the map $f$ from $X$ to $D$ defined by $f(U)=\{0\}$ and $f\left(U^{c}\right)=\{1\}$ is a continuous map. Now, we have $f(U)$ is an open neighborhood of $f(x)$ such that $f(y) \notin f(U)$, as required.

Using Theorem 6, we can deduce the next corollary.

Corollary 2. We have the following equivalences:

$$
F T_{(0, D)} \Leftrightarrow F T_{(1, D)} \Leftrightarrow F T_{(2, D)} .
$$

Now, we denote by $S$ the Sierpinski space $S=\{0,1\}$ with the topology $\tau_{S}=\{\varnothing, S,\{1\}\}$.

Theorem 7. Let $(X, \tau)$ be a topological space such that $X$ is not a singleton. Then, the next statements are identical:

(1) $X$ is an $F T_{(0, S)}$-space

(2) $X$ is a $T_{0}$ space

Proof

$(1) \Rightarrow(2)$ : by Proposition 4 .

$(2) \Rightarrow(1)$ : suppose $X$ is a $T_{0}$-space. Let $x \neq y \in X$. Then, there is open set $U$ containing $x$ or $y$ but not both. Say, $x \in U$. Now, the map $f$ from $X$ to $S$ defined by $f(U)=$ $\{1\}$ and $f\left(U^{c}\right)=\{0\}$ is continuous and $\{1\}$ is an open subset containing $f(x)$ and not containing $f(y)$. This fact completes the proof.

Remark 2. There is no topological space which is $F T_{(1, S)}$, so that there is no one which is $F T_{(2, S)}$.

We denote by $\mathcal{O}(a)$ the set of all open neighborhoods of $a$ in a topological space $(X, \tau)$.

Theorem 8. The next statements are identical:

(1) $S$ is $F T_{(0, Y)}$

(2) $\exists a, b \in(Y, \theta)$ such that $\mathcal{O}(b) \subset \mathcal{O}(a)$ strictly

Proof

$(1) \Rightarrow(2)$ : let $f$ be a continuous map from $S$ to $Y$ and $U \in \theta$ such that $f(1) \in U$ and $f(0) \notin U$. Then, $\mathcal{O}(f(0)) \subset \mathcal{O}(f(1))$ strictly.

$(2) \Rightarrow(1)$ : let $a, b \in Y$ such that $\mathcal{O}(b) \subset \mathcal{O}(a)$ strictly. We define the map $f$ from $S$ to $Y$ by $f(1)=a$ and $f(0)=b$. On the one hand, $f$ is continuous because every open set containing $b$ contain also $a$. On the other hand, since the inclusion $\mathcal{O}(b) \subset \mathcal{O}(a)$ is strict, there exists an open neighborhood of $f(1)$ which does not contain $f(0)$; hence, we deduce that $S$ is $F T_{(0, Y)}$.
Corollary 3. If $S$ is $F T_{(0, Y)}$, then $Y$ is not a $T_{1}$-space.

Let $(X, \tau)$ be a Functionally Hausdorff space. Then, $(X, \tau)$ can be seen as $F T_{(2, \mathbb{R})}$. What about a topological space $(Y, \theta)$ such that $\mathbb{R}$ is $F T_{(2, Y)}$. The following proposition gives a sufficient condition for such topological space.

Proposition 7. Let $(Y, \theta)$ be a topological space. If $(Y, \theta)$ satisfies the following conditions:

$\exists a, b, c \in Y, U \in \mathcal{O}(a)$ and $V \in \mathcal{O}(b)$ such that $U \cap V=\varnothing$ and $\mathcal{O}(c) \subseteq \mathcal{O}(a) \cap \mathcal{O}(b)$.

Then, $\mathbb{R}$ is $F T_{(2, Y)}$.

Proof. Let $x<y \in \mathbb{R}$ and $f$ be the map from $\mathbb{R}$ to $Y$ defined by $f(]-\infty,(x+y) / 2[)=\{a\}, \quad f(](x+y) / 2,-\infty[)=\{b\}$ and $f((x+y) / 2)=c$. Then, $f$ satisfies the third separation axioms in Definition 7.

The following example shows that the condition in the previous proposition is sufficient but not necessary for a topological space $(Y, \theta)$ such that $\mathbb{R}$ is $F T_{(2, Y)}$.

Example 3. Let $Y=\{1,2,3\}$ with the topology $\theta=\{\varnothing,\{1\},\{2\},\{1,2\}, Y\}$. Then, $\mathbb{R}$ is $F T_{(2, Y)}$. Note that $\mathbb{R}$ does not satisfy the condition given in the above proposition because for any three elements $a=1, b=2, c=3$ of $\mathbb{R}$, we have $\mathcal{O}(1) \subseteq \mathcal{O}(2) \cap \mathcal{O}(3), \quad \mathcal{O}(2) \subseteq \mathcal{O}(1) \cap \mathcal{O}(3)$, and $\mathcal{O}$ $(3) \subseteq \mathcal{O}(1) \cap \mathcal{O}(2)$. On the other hand, $\mathbb{R}$ is $F T_{(2, \mathbb{R})}$.

It is natural now to pose the question about the necessary and sufficient condition for a topological space $(Y, \theta)$ such that $\mathbb{R}$ is $F T_{(2, Y)}$. The following theorem answers this question.

Theorem 9. Let $(Y, \theta)$ be a topological space. Then, the next properties are identical:

(1) $\mathbb{R}$ is $F T_{(2, Y)}$

(2) $\exists U, V \in \theta$ and a continuous map $g$ from $\mathbb{R}$ to $Y$ such that $U \cap V=\varnothing, g(0) \in U$ and $g(1) \in V$

Proof. The first implication is trivial by the definition of FT $_{(2, Y)}$.

Conversely, if $x$ and $y$ are two distinct real numbers, then there exists an isomorphism $h$ from $\mathbb{R}$ to itself satisfying $h(x)=0$ and $h(y)=1$. So that the map $f=g \circ h$ is continuous such that $f(x) \in U$ and $f(y) \in V$. This completes the proof.

It is well known that any map from a discrete topological space $\left(X, \tau_{d}\right)$ to any topological space $(Y, \theta)$ is continuous. We benefit from this fact to establish the following results.

Proposition 8. Let $(Y, \theta)$ be a topological space and $|X| \geq 2$. Then, the following properties are equivalent:

(1) $\left(X, \tau_{d}\right)$ is $F_{(0, Y)}$

(2) $|\theta| \geq 3$ 
Proof

$(1) \Rightarrow(2)$ : this direction is obvious.

(2) $\Rightarrow(1)$ : let $a \neq b \in X$ and $O \in(\theta /\{Y, \varnothing\})$. We define a map (continuous) $f$ from $X$ to $Y$ by $f(a) \in O$ and $f(x) \notin O$ for all $x \neq a$. Hence, $(X, \tau)$ is $F T_{(0, Y)}$, as required.

Proposition 9. Let $(Y, \theta)$ be a topological space and $|X| \geq 2$. Then, the following properties are equivalent:

(1) $\left(X, \tau_{d}\right)$ is $F T_{(1, Y)}$

(2) There exist two distinct points $y, z \in Y$ such that the topology of the subspace $A=\{y, z\}$ is the discrete topology

Proof. Suppose that $X$ is $F T_{(1, Y)}$ and let $a \neq b \in X$. Then, there exists $U, V \in \theta$ such that $f(x) \in(U / V)$ and $f(y) \in(V / U)$. It is clear that the subspace $A=\{f(a), f(b)\}$ is equipped by the discrete topology.

Conversely, it is sufficient, for every two distinct points $a, b$ in $X$, a map $f$ such that $f(a)=y$ and $f(b)=z$.

Theorem 10. Let $(Y, \theta)$ be a topological space and $|X| \geq 2$. Then, the following properties are equivalent:

(1) $\left(X, \tau_{d}\right)$ is $F T_{(2, Y)}$

(2) There exist $O_{1}, O_{2} \in \theta$ such that $O_{1} \cap O_{2}=\varnothing$

Proof

(1) $\Rightarrow(2)$ : this direction follows from 3 of Definition 7 .

(2) $\Rightarrow(1)$ : let $a \neq b \in X$. We define a (continuous) map $f$ from $X$ to $Y$ such that $f(a) \in O_{1}$ and $f(x) \notin O_{2}$ for all $x \neq a$. Hence, we obtain that $\left(X, \tau_{d}\right)$ is $F T_{(2, Y)}$, as required.

Corollary 4. If $(Y, \theta)$ is disconnected topological space, then $\left(X, \tau_{d}\right)$ is $F T_{(i, Y)}$ for each $i \in\{0,1,2\}$.

\section{Conclusion}

This manuscript contributes to the area of separation axioms. We have applied continuous maps to introduce the concepts of functionally $T_{i}$ spaces for $i=0,1,2$. We have elucidated the relationships between them and clarified that they are weaker than $T_{i}$ spaces, see Proposition 3 and Proposition 4. Also, we have investigated some results associated them with product spaces and categories. Moreover, their behaviours with some special spaces such as discrete and Sierpinski spaces have been studied.

In the upcoming work, we plan to explore functionally regular and functionally normal spaces. Also, we generalize the presented concepts presented in this manuscript using somewhere dense sets and SD-continuous maps given in $[7,16,17]$

\section{Data Availability}

No data were used to support this study.

\section{Conflicts of Interest}

The authors declare no conflicts of interest.

\section{References}

[1] E.-S. A. Abo-Tabl, "On links between rough sets and digital topology," Applied Mathematics, vol. 5, no. 6, pp. 941-948, 2014.

[2] A. S. Salama, "Topological solution of missing attribute values problem in incomplete information tables," Information Sciences, vol. 180, no. 5, pp. 631-639, 2010.

[3] W. Zhu, "Topological approaches to covering rough sets," Information Sciences, vol. 177, no. 6, pp. 1499-1508, 2007.

[4] Z. Ercan, "A characterization of completely Hausdorff spaces," Mathematical Proceedings of the Royal Irish Academy, vol. 117A, no. 1, pp. 1-4, 2017.

[5] S. Lazaar, "On functionally hausdorff spaces," Missouri Journal of Mathematical Sciences, vol. 25, no. 1, pp. 88-97, 2013.

[6] H. L. Lord, "Functionally hausdorff spaces," Cahier de topologie et géométrie différentielle catégoriques, vol. 30-3, pp. 247-256, 1989.

[7] T. M. Al-shami, "Somewhere dense sets and $S T_{1}$-spaces," Punjab University Journal of Mathematics, vol. 49, no. 2, pp. 101-111, 2017.

[8] M. Abbassi, S. Lazaar, and A. Mhemdi, " $T_{0}$-reflection and some separation axioms in PreTop," Filomat, vol. 32, no. 9, pp. 3289-3296, 2018.

[9] T. M. Al-shami and M. Abo-Elhamayel, "Novel class of ordered separation axioms using limit points," Applied Mathematics \& Information Sciences, vol. 14, no. 6, pp. 1103-1111, 2020.

[10] S. Lazaar and A. Mhemdi, "On some properties of $T_{0}$ ordered reflection," Applied General Topology, vol. 15, 2014.

[11] G. Mirhosseinkhani, "Quasihomeomorphisms and meetsemilattice equivalences of generalized topological spaces," Acta Mathematica Hungarica, vol. 147, no. 2, pp. 272-285, 2015.

[12] K. Belaid, O. Echi, and S. Lazaar, " $T(\alpha, \beta)$-spaces and the Wallman compactification," International Journal of Mathematics and Mathematical Sciences, vol. 68, pp. 3717-3735, 2004.

[13] A. Alpers, "Digital topology: regular sets and root images of the cross median filter," Journal of Mathematical Imaging and Vision, vol. 17, no. 1, pp. 7-14, 2002.

[14] S.-E. Han, "Hereditary properties of semi-separation axioms and their applications," Filomat, vol. 32, no. 13, pp. 4689-4700, 2018.

[15] J. R. Munkres, Topology, Prentice-Hall, Hoboken, NJ, USA, 1975.

[16] T. M. Al-shami and T. Noiri, "More notions and mappings via somewhere dense sets," Afrika Matematika, vol. 30, no. 7-8, pp. 1011-1024, 2019.

[17] T. M. Al-shami and T. Noiri, "Compactness and Lindelöfness using somewhere dense and cs-dense sets," Novi Sad Journal of Mathematics, 2021. 